\title{
3D Game Graphic Complexity Effects on Motion-Onset Visual Evoked Potentials
}

Beveridge, R., Marshall, D., Wilson, S., \& Coyle, D. (2015). 3D Game Graphic Complexity Effects on MotionOnset Visual Evoked Potentials. In Unknown Host Publication (pp. 139-147). Global Science and Technology Forum. https://doi.org/10.5176/2251-1679_CGAT15.39

Link to publication record in Ulster University Research Portal

\author{
Published in: \\ Unknown Host Publication
}

Publication Status:

Published (in print/issue): 01/01/2015

DOI:

10.5176/2251-1679_CGAT15.39

\section{Document Version}

Publisher's PDF, also known as Version of record

\section{General rights}

Copyright for the publications made accessible via Ulster University's Research Portal is retained by the author(s) and / or other copyright owners and it is a condition of accessing these publications that users recognise and abide by the legal requirements associated with these rights.

\section{Take down policy}

The Research Portal is Ulster University's institutional repository that provides access to Ulster's research outputs. Every effort has been made to ensure that content in the Research Portal does not infringe any person's rights, or applicable UK laws. If you discover content in the Research Portal that you believe breaches copyright or violates any law, please contact pure-support@ulster.ac.uk. 


\title{
3D Game Graphic Complexity Effects on Motion-Onset Visual Evoked Potentials
}

\author{
Ryan Beveridge, David Marshall, Shane Wilson and Damien Coyle \\ Intelligent Systems Research Centre, University of Ulster, Derry, Northern Ireland, U.K. \\ Beveridge-R@email.ulster.ac.uk, Marshall-D3@email.ulster.ac.uk, s.wilson@ulster.ac.uk and dh.coyle@ulster.ac.uk
}

\begin{abstract}
Motion Onset Visually Evoked Potentials (mVEPs) have the advantage of being an elegant and less visual fatiguing stimuli than that of other VEPs such as the steady state VEP (SSVEP) or P300 and therefore may be apposite for use in movement-free brain-controlled computer games using braincomputer interface (BCI) technology. To investigate the effects of 3-Dimensional (3D) graphic variations on mVEPs, we present in this pilot study a set of five hypothetical game levels with differing graphics, each with increasing visual complexity, in which the user/player must attend to one of five mVEP inducing stimuli. The mVEP based on-screen virtual buttons involves a leftward motion lasting 140 milliseconds to elicit a response from the dorsal pathway. This pilot study focused on offline classification results. BCI classification accuracy results for separating target vs. non target $\mathrm{mVEP}$ stimuli ( 2 class) as well as classifying target stimuli among the five stimuli (5 class) are compared for each variation in graphic complexity. The results of the study show a trend indicating the classification accuracy is inversely proportional to graphic complexity however the difference in BCI classification accuracy for each level of complexity are not significant $(>70 \%, p>0.05)$. The results are encouraging, suggesting that the use of 3D graphics of varied complexity is possible when using mVEP based BCI as a control strategy.
\end{abstract}

Keywords - Brain-Computer Interface (BCI), Motion Onset Visually Evoked Potentials (mVEP), Electroencephalography (EEG), Gaming, 3D, Graphics, Visual

\section{INTRODUCTION}

Research into the application of braincomputer interface (BCI) [1] as a control scheme for computer games has increased dramatically in recent years and is reflected in the number of published papers on the topic [2]. The notion that one can control a video game without the need for muscle control i.e. "movement-free" may provide novel gaming experiences and new forms of immersion for gamers. It will also allow physically impaired users such as those suffering from Amyotrophic Lateral Sclerosis (ALS), spinal cord injury or brainstem stroke, access to video gaming technology providing them with additional forms of entertainment. An Electroencephalography (EEG) based BCI provides a method of communication which involves reading brain activity non-invasively via a set of electrical sensors placed directly on the user's scalp.

Most BCI games to date have not appealed to traditional gamers due largely to the rudimentary graphics employed. To date the graphical richness of BCI games has been compromised in favour of gaining BCI system accuracy. In traditional games rich graphical complexity is merely part of the gameplay experience and helps the player feel immersed in gameplay but in a BCI controlled game, rich graphics may hinder the accuracy of the $\mathrm{BCI}$ system due to their motion, depth or use of flashing imagery.

Limits in the accuracy of interaction and the level of latency in BCI games to date are suspected issues preventing widespread adoption of the technology. This is due to the speed at which the $\mathrm{BCI}$ system can analyse, interpret and convert the ongoing EEG signals into useful commands for a video game to recognise and execute. This caveat limits the players' interaction options within BCI games and may not promote BCI technology in the best possible light [3]. Another reason why adoption of BCI as a control method has been slow is the need for electrodes to be mounted on the users scalp. This need for electrodes introduces other factors into the BCI system such as higher costs and setup times for the hardware. However, recent technological improvements in hardware have led to advances such as less expensive, dry type electrodes which 
have lowered both prices and setup times for BCI hardware [4].

In most studies, $\mathrm{BCI}$ games have been used to test the feasibility of BCI system or control strategy, maintain the interest of the user whilst they learn to operate a BCI and to retrieve test results rather than as a form of entertainment in their own right. The disadvantage of only creating a game for testing purposes is that the game does not appeal to the wider gaming population and hence the perception of BCI among the gaming community is perhaps not as positive as could be to date. The limited scope, controller input and limited visual appeal in most BCI games requires improvement. This paper provides a preliminary study to address one of the above mentioned issues relating to BCI gaming, namely assessing the effect of graphic complexity in games on brain-computer interface (BCI) performance.

This study focuses on testing the Motion onset Visual Evoked Potential (mVEP) based BCI control strategy (see section 2.1 below) for an action game. The action genre is the most popular game genre used in BCI [2] although there are only a limited number of studies employing $\mathrm{mVEP}$ as a control method [5][6][7]. mVEP offers a less visually fatiguing stimulus than other BCI control methods such as P300 and steady state visually evoked potentials (SSVEP) (summarised below) due its use of motion rather than flashing imagery and it can also be used with low luminance values. mVEP also has the advantage that little user training is required for its successful use. There are a number of control strategies available to designers of a BCI video game and each comes with its own (dis)advantages. A short overview of the most common BCI strategies used are provided in the following sections.

Options for game control available to BCI game designers include P300 which uses flashing stimuli to elicit an Event Related Potential (ERP) response in the visual cortex and can be seen as a large positive peak in the ongoing EEG signal 250500 milliseconds after an evoked stimulus [8][9]. Steady state visual evoked potentials also make use of flashing stimuli to elicit an ERP such as the reverse chequerboard pattern (chequerboard patterns alternating at a specific frequency) which elicits a response in the visual cortex and can be seen as a large positive peak at specific frequencies in the ongoing EEG signal [10][11]. Motor Imagery (MI) is a BCI control method which uses cortical changes known as Event Related Synchronisation (ERS) and Event Related Desynchronisation (ERD) in the motor cortex of the brain when the user imagines or performs body movements such as imagined movement of the hands, feet or tongue [12][13].

Motion Onset Visual Evoked Potentials (mVEP) involves the use of moving imagery to elicit a response from the dorsal pathway [7]. A typical mVEP interface as used in [5][6][7] consists of a set of on-screen virtual buttons which are rectangular in shape (Fig. 1). The red vertical line inside one of the buttons will start moving (motion onset) and move from the right hand side of the button in a continuous motion to the left hand side of the button and then disappear (motion offset). This motion of the vertical line will happen in each of the virtual buttons in turn and when a user concentrates on their required button, a response will be elicited in the ongoing EEG signal when the user has recognised his/her required choice. It is the onset of motion in each of the buttons which stimulates a response from the dorsal pathway of the brain and can be seen in the ongoing EEG signal as an N2 peak with a latency of $160-200 \mathrm{~ms}$, followed by a P2 peak with a latency of around $240 \mathrm{~ms}$. It is these clear and robust EEG features which highlight $\mathrm{mVEP}$ as a suitable method for BCI control.

An mVEP BCI system can operate at low luminance levels, which provides ideal properties for gaming use as the mVEP controls may be presented to the user without affecting the in game imagery such as graphics, feedback text and moving imagery etc. mVEP also has the advantage of low visual fatigue which is important because in the case of gaming there are already multiple on-screen items and game characteristics that exhibit properties which can cause visual fatigue such as fast moving, flashing imagery.

Also, very little user training is required to successfully use an mVEP BCI and the importance of this becomes clear when making games available to the commercial market where users may not be willing to train how to use a BCI system out of a laboratory setting.

The accuracy of an mVEP based BCI system can be high as can be seen in [5] where the authors reported $>90 \%$ accuracies for all 15 subjects tested after just ten trails. These accuracies were reported using a five class (five mVEP buttons) BCI setup. As with other BCI control methods however, latency still remains an issue in $\mathrm{mVEP}$.

The hypothetical game chosen for this study used a basic capsule shaped third person character and increasingly complex graphics over the course of four game levels. The purpose of the study was to investigate if the mVEP accuracies were affected 
by the increasingly complex $3 \mathrm{D}$ and full colour graphics. It was hypothesised that as the graphical complexity increases with added colours, items or speed, the mVEP accuracy would decrease due to increased visual distraction and attractions the user is likely to attend to or ignore.

The classification accuracy from mVEP stimulated using five on screen stimuli for five different levels of graphical complexity, namely, basic training, character movement, characters speed with changing coloured background, in game pickup items at side of screen and in game pickup items placed on characters path to dodge was assessed.

Section 2 details the methodology undertaken for this study. Section 3 presents the methods used to calculate the accuracies achieved from each subject. Section 4 discusses the results from the study. Section 5 is the discussion section which details the limitations of this study and Section 6 concludes the paper.

\section{METHODOLOGY}

\subsection{Paradigm}

The paradigm and game graphics presentation was developed using the Unity 3D [14] game development engine. A mVEP paradigm with a five button layout is placed at the top of the screen, arranged in a horizontal configuration.

A number placed at the top of each mVEP button differentiated the buttons from each other and also acted as the indicator of which button the user should focus their attention by changing colour from black to red. The user was instructed to focus their attention on the moving line of the red button (Fig. 1 shows the training level with the ' 400 ' button as currently active while ' 200 ' is the users current target (shown in red). The motion of each mVEP button consisted of the red coloured vertical line moving horizontally from the right hand side to the left hand side of the rectangle.

The timing protocol for this study followed closely to that of [7]. A game level consisted of either 150 (for the first three subjects) or 300 (for the remaining seven subjects) trials. Each level lasted 960 seconds, during which, each mVEP button will have been activated 60 times. During a trial, each of the five buttons is active five times. Each button is highlighted in turn. In order to avoid user habituation, each of the buttons motion are activated in random order. The Stimulus Onset Asynchrony (SOA) between each button activation is $200 \mathrm{~ms}$. The motion of the vertical line moving from right to left in each button lasts $140 \mathrm{~ms}$ and the time between each button activation is $60 \mathrm{~ms}$. A diagram of the timing protocol used is shown in (Fig. 2).

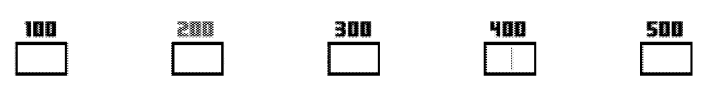

Figure 1. mVEP Training Level - no graphics.

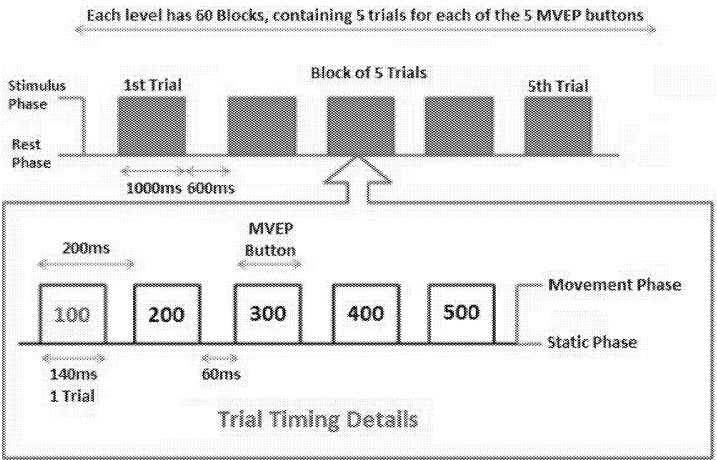

Figure 2. mVEP Timing protocol used in this study.
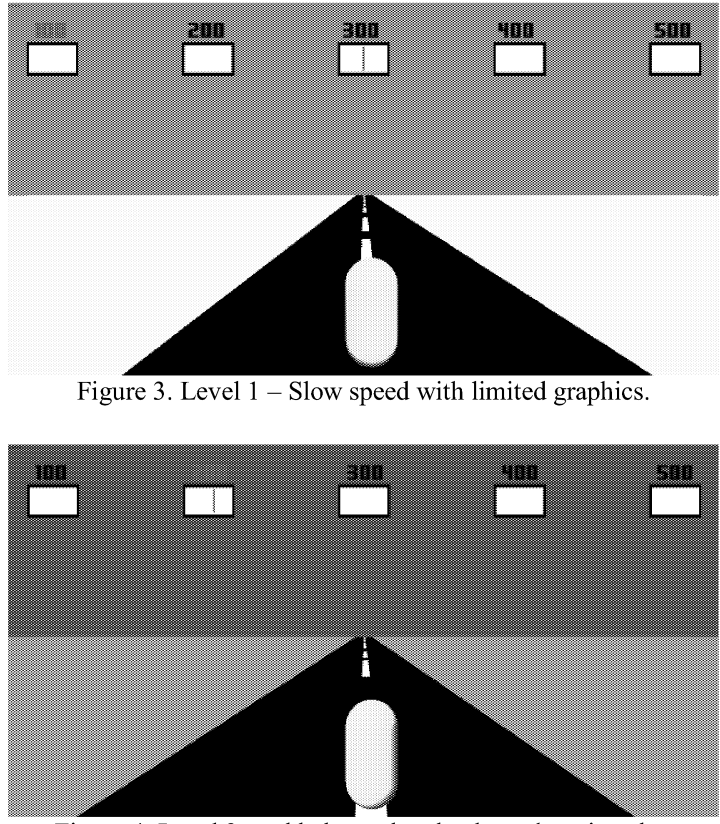

Figure 4. Level 2 - added speed and colour changing sky. 


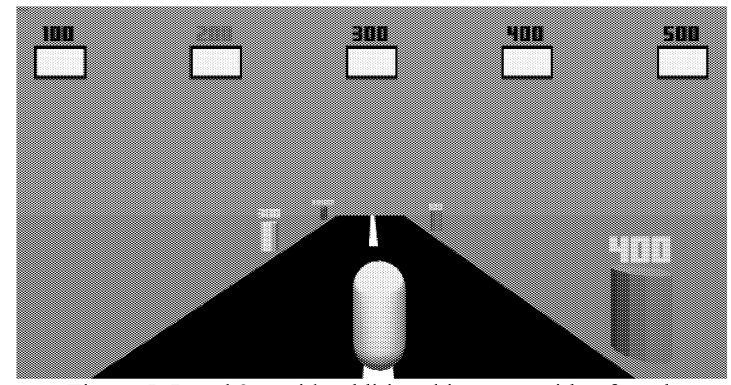

Figure 5. Level 3 - with additional items on side of road.

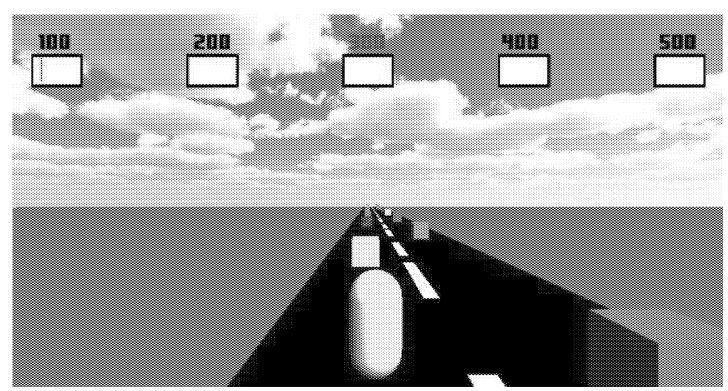

Figure 6. Level 4 - obstacles to avoid and realistic sky.

The paradigm commenced with a training level using a plain white background with the five mVEP buttons arranged horizontally in the centre of the screen (Fig. 1). The training level was followed by Level 1 (Fig. 3), which showed a character constantly moving in the forward direction with basic textured road surface, sky and grass. Level 2 (Fig. 4) showed the character moving in the forward direction with the addition of more speed and the colour of the skyline constantly changing. Level 3 (Fig. 5) was as level 1 with the addition of barrel shaped objects placed at the side of the road with a player score attached to each. Level 4 (Fig. 6) was as level 3 with an added realistic sky background, but this time the items were placed on the road and the character automatically avoided these so in this case the character moved horizontally across the screen to add further distraction and complexity. The setup of the four games presentation levels resulted in a gradual increase in visual complexity as the levels progressed. To assess the accuracy of the results according to graphical complexity, rather than gameplay experience, the user had no control over the character and there was no real-time feedback on the user's selection performance i.e., all the character movements were automatically controlled and the user's only task was to focus on the required mVEP stimuli. The only graphical user interface information other than the mVEP button arrangement was in Level 3 where each barrel had scoring information attached. The scoring information only served as a further visual distraction and required no input from the user. This was added into the level to see how the on screen scoring information affected the mVEP accuracy.

\subsection{Data Acquisition}

Two female and eight male subjects took part in this study. Two of these subjects had previous $\mathrm{BCI}$ experience using $\mathrm{mVEP}$ and the other eight were BCI naive. Each subject was involved in a single recording session with the training plus four game levels assessed in the session. An EEG cap was placed on the participants head and electrodes were placed over occipital areas using a 12 channel montage (Fig. 7) according to the international 1020 system of electrode placement [15]. The left mastoid acted as ground and $\mathrm{FPz}$ as the reference voltage. Recording took place in an electrostatically shielded/acoustically insulated room and participants were seated on a comfortable chair placed $50 \mathrm{~cm}$ in front of an LCD computer monitor $56 \mathrm{~cm}$ (Width $47.7 \mathrm{~cm}$ and Height $29.8 \mathrm{~cm}$ ) in size and a refresh rate of $60 \mathrm{~Hz}$.

EEG data was collected using a g.BSamp amplifier [16], g.Gammasys active electrode system [17] connected to an Easycap electrode cap [18] and Matlab Simulink [19]. As Unity 3D presented each visual cue to the user, a stimulus identifier relating to each mVEP stimulus along with timing information were sent to Simulink from Unity 3D using the UDP (User Datagram Protocol) transmission convention. The stimulus trigger information and EEG signals are co-registered in Simulink.

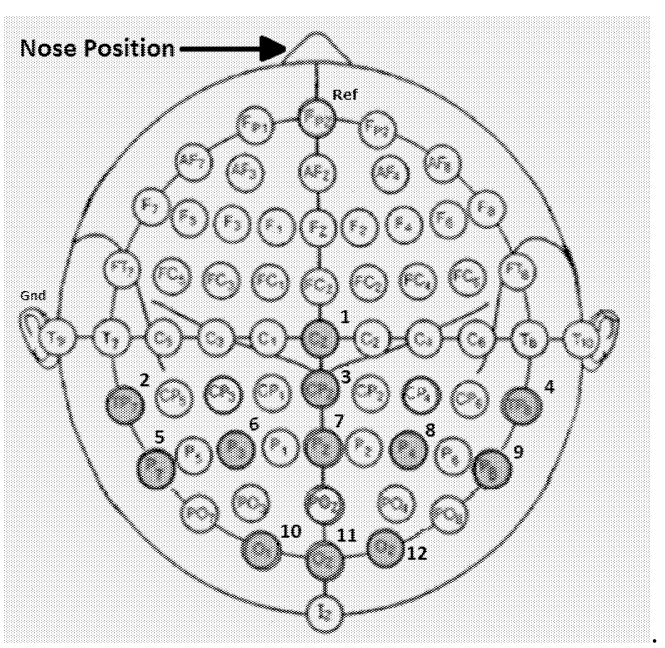

Figure 7. 12 Channel montage used in the study with FPz as reference voltage and left mastoid as ground. 


\section{DATA ANALYSIS}

\subsection{Data pre-processing Methods}

A total of 300 trials per level (each stimuli was a target for 60 trials) were recorded from each subject. Data epochs were derived in association with each motion onset stimulus, beginning $200 \mathrm{~ms}$ prior to the motion onset and lasting for $1200 \mathrm{~ms}$. All single trials were baseline corrected with respect to the mean voltage over the $200 \mathrm{~ms}$ preceding motion onset. Data were digitally filtered using a low-pass Butterworth filter (order 5, with cut-off at $10 \mathrm{~Hz}$ ) and subsequently resampled at $20 \mathrm{~Hz}$. Features were extracted between $100 \mathrm{~ms}$ and $500 \mathrm{~ms}$ (the epoch which normally contains the most reactive mVEP components e.g. N200, P300 and N400) post stimulus yielding nine features for each channel. Data were averaged over five trials yielding twelve features vectors per stimulus for each level. Data was split into target vs. non target where for each non target feature vector five randomly selected non-target trials were used.

Since mVEP is time locked and phase locked to the motion onset stimulus, mVEP induced from the motion stimuli could be obtained through the above simple averaging procedure [5].

\subsection{Channel Selection}

A Linear Discriminant Analysis (LDA) classifier was trained to discriminate target vs. non target feature vectors extracted from single channels in a Leave One Out (LOO) cross validation on $50 \%$ of the data (the remaining $50 \%$ was held for final testing). For each of the twelve channels the average LOO classification accuracy (LOO-CA) was determined and channels were ranked by accuracy. The most common three channels across all subjects consisted of O1, P7 and TP7. The top three ranked channels were concatenated to form a new feature vector (27 features per vector) and a further LOO cross validation was performed. The results of this are reported as LOO-CA3. A single trial test of target vs. non target is also applied on the training data (Target vs. Non Target - Single Trial).

\section{3. mVEP Classification - 5 Class}

Using all the training data $(50 \%$ of data) a new LDA classifier is produced to classify target vs. non target data. To classify individual symbols in a single trial test each feature vector associated with each stimulus in a trial is classified as either target or non-target. The LDA classifier produced a distance value, $D$, reflecting the distance from the hyper plane separating target and non-target features $(D>0$ for target and $D<0$ for non-target). The vector that produces the maximum distance value is selected as the classified stimulus (in some cases non-target data produces a $D>0$, however the value of $D$ is normally maximal among the target stimulus i.e., the stimulus on which the user is focused). Single trial results for five class are reported for the training data and then the setup is applied on the remaining $50 \%$ of the data, unseen testing data.

A second set of tests were performed which involved applying the classifier trained on the training level on the remaining four games levels (as opposed to retraining the classifier for each level). The evaluation reflects the performances that may be attainable in an online situation where the user may play the games within a single training run, but the classifier is only trained once at the beginning of the session.

Offline analysis was performed using customised MATLAB code along with the BioSig [20] and LIBSVM [21] toolboxes.

\section{RESULTS}

\subsection{Offline Testing}

Data from all subjects were analysed level by level for all five levels (training plus the game levels 1-4) with results presented as LOO-CA3, target vs. non target single trial (training), single trial 5 class (training) and single trial 5 class (testing). Fig. 8 shows the average LOO-CA3 result for all ten subjects across the five game levels. As can be seen in the graph, there is a slight downwards trend in accuracy as the game levels increase in visual complexity with level 4 accuracy being lower than the training accuracy as well as the level 1 accuracy. A single factor Analysis of Variance (ANOVA) test was conducted for the LOO-CA3 analysis and suggests the difference in accuracy for each level are not statistically significant $(p>0.05)$.

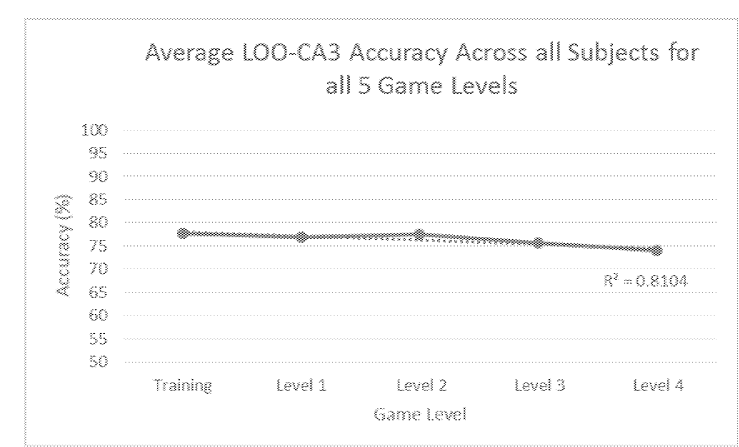

Figure 8. Graph to show the average LOO-CA3 accuracy across all 5 game levels. $\mathrm{R}^{2}=0.81$. 
Fig. 9 shows the results for the target vs. non target Single Trial (training) analysis method. Here, as can be seen in the graph, a more pronounced downwards trend in accuracy. As the game levels progress, the accuracy decreases in a linear fashion. However, an ANOVA test reveals the differences in accuracy are insignificant $(p=0.79)$.

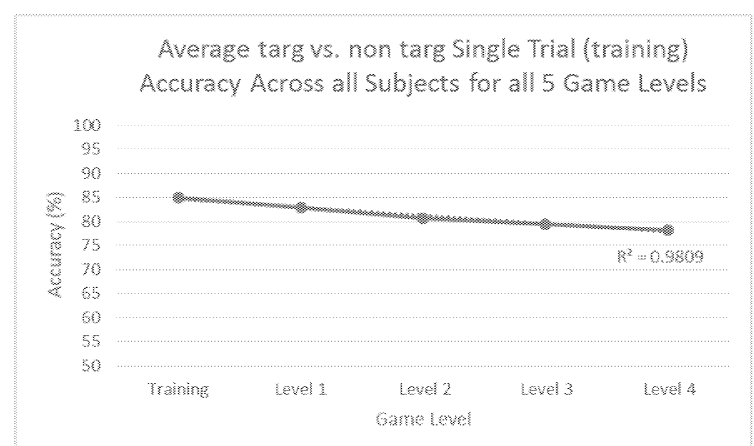

Figure 9. Graph to show the mean Target vs. non Target Single Trial (training) accuracy across all 5 game levels. $R^{2}=0.98$.

Fig. 10 shows the results for the Single Trial 5 Class (training) analysis method. Again, there is a clear downwards trend in accuracy across the five game levels, beginning with the training level and gradually decreasing linearly until level 4 , again insignificant differences $(p=0.62)$.

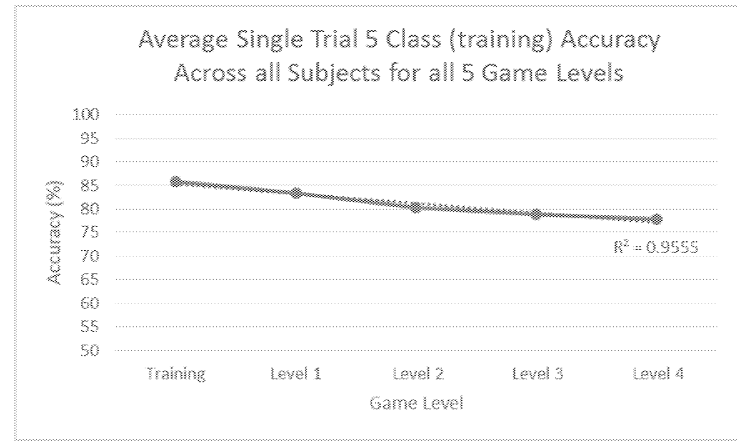

Figure 10. Graph to show the mean Single Trial 5 Class (training) accuracy across all 5 game levels. $\mathrm{R}^{2}=0.96$.

The final analysis method to be analysed was Single Trial 5 Class (testing). Only seven of the ten subjects' data were analysed using this method as there were only 150 trials per level for the first three subjects to participate in the experiments. For the remaining seven subjects there were 300 trials recorded in order to leave out $50 \%$ of the data to test the system accuracy.

Fig. 11 shows the results for the Single Trial 5 Class (testing) analysis method. As can be seen in the graph, again there is a downwards trend in accuracy. The levels gradually decrease from the training level to level 3 , but a slight increase can be seen again by level $4(p=0.78)$.

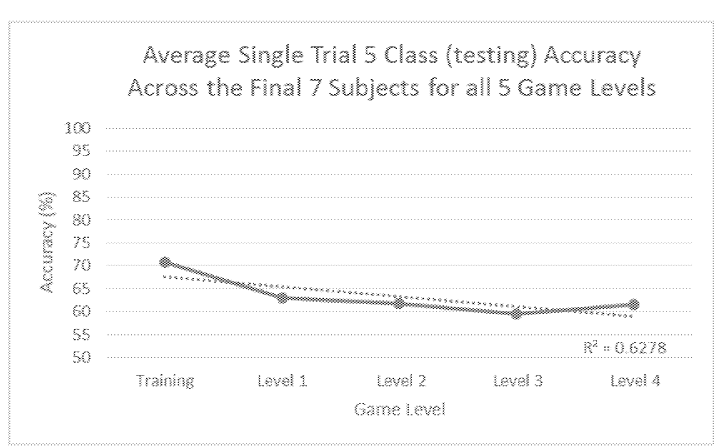

Figure 11. Graph to show the mean Single Trial 5 Class (testing) accuracy for the final 7 subjects across 5 game levels. $\mathrm{R}^{2}=0.63$.

From analysis of the offline results, it can be seen that there are clear downwards trends in all metrics of performance as the game levels graphical complexities increase, yet none of these trends been seen to show significant difference in performance due to graphical complexity.

\subsection{Cross Level Testing}

In order to test the transfer of the classifier from training data to each of the four levels, the data acquired from each of the subjects' initial training sessions was used to determine the system parameters and classifier and then these were applied onto the data acquired from each of the four different levels of complexity.

Fig. 12 shows the average results across all subjects for cross level tests for all four game levels. It is clear that the accuracy is diminished when applying a classifier from training to each of the levels as opposed to retraining the classifier on a portion of the data from each level. We can see from the graph that there is a sharp decrease in accuracy between the first two game levels and the final two game levels. A further point to note is the slight increase in level 2 from level 1 and the increase in level 4 from level 3. 


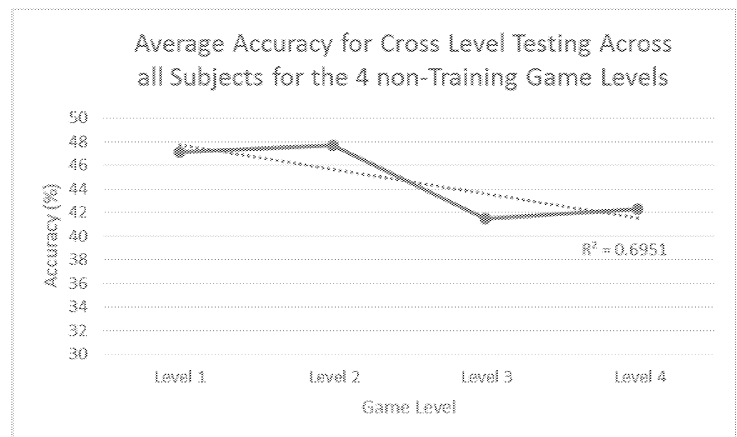

Figure 12. Graph to show the mean cross level accuracy for all subjects across the 4 non-training game levels. $\mathrm{R}^{2}=0.70$.

From analysis of the cross level results, it can be concluded that towards the end of the BCI session and as the graphical complexity increased, the accuracies decreased. The ANOVA results for the cross level testing also show that the differences are not significant $(p=0.86)$.

\subsection{Individual Subject Performances}

TABLE I. AVERAGE RESULTS (\% ACCURACY) ACROSS ALL SUBJECTS FOR THE 4 ANALYSIS METHODS.

\begin{tabular}{|c|c|c|c|c|}
\hline & \multicolumn{3}{|c|}{ 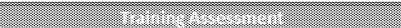 } & \multirow{3}{*}{ Five Class } \\
\hline & \multicolumn{2}{|c|}{ Target vs Non Target } & Five Class & \\
\hline & $1830=8.8$ & $2.125=12$ & $2.0=1.2$ & \\
\hline S1 & 76.4 & 89.5 & 90.7 & 74.9 \\
\hline \$2 & 74.5 & 76.9 & 75.5 & 67.6 \\
\hline 53 & 67.3 & 70.1 & 68.9 & 48.8 \\
\hline 54 & 74.8 & 71.6 & 72.0 & $n / a$ \\
\hline s5 & 89.5 & 98.1 & 95.9 & $\mathrm{n} / \mathrm{a}$ \\
\hline 56 & 77.6 & 82.1 & 83.2 & $\mathrm{n} / \mathrm{a}$ \\
\hline 57 & 71,5 & 75.6 & 77.1 & 56.1 \\
\hline 58 & 78.1 & 85.5 & 86.9 & 74.1 \\
\hline s9 & 66.9 & 65.6 & 65.1 & 41.3 \\
\hline S10 & 87,2 & 97.9 & 97.5 & 80.4 \\
\hline Average & 76.4 & 81.3 & 81.3 & 63.3 \\
\hline
\end{tabular}

Table I shows the average accuracies for all subjects for the four analysis methods. Subject S5 and S10 achieved consistently high accuracies throughout the trials. Subject S5 performed best for the analysis methods LOO-CA3 and Target vs. non-target Single Trial (training) and subject S10 performed best on analysis methods single trial five class (training) and single trial 5 class (testing). Perhaps if subject S5 had participated in 300 trials instead of 150, their accuracies may have been improved for the final two analysis methods.

The worst performing subject was S9 who achieved the lowest accuracies across all performance metrics. The final method Single Trial 5 Class (testing) provided the lowest accuracy for subject $\mathrm{S} 9$ at $41.3 \%$. It is interesting to note that this subject (S9) reported that they were physically fatigued during the session due to partaking in physical exercise one hour prior to taking part in the
BCI session. Perhaps a further session using the same subject in a more relaxed, less fatigued state may produce greater accuracies.

\section{DISCUSSION}

mVEP is a relatively new type of VEP to be applied in BCI and so far has not been widely studied in this context with only a few studies investigating $\mathrm{mVEP}$ controlled computer games [7]. Gao et al [5] published the first mVEP based BCI study. In that paper, they investigated an on screen virtual five button arrangement based on the brief horizontal motion of a vertical line. It is the brief motion of this vertical line which invokes a response from the dorsal pathway of the brain. This study focused on the use of the mVEP paradigm as it provides the BCI user with a more aesthetically pleasing visual stimulus while maintaining low visual fatigue for the user. This makes mVEP a more desirable choice for use in BCI games as some games are played for prolonged periods of time and already exhibit visual complexities such as flashing imagery and fast moving backgrounds.

In the current study, we have used a similar mVEP button design to that of Gao et al and studied mVEP classification in the context of a 3D graphics presentation to determine the effects of moving and changing 3D graphics on the classification accuracy of mVEPs. The results show that as the graphical complexity of the game levels increase with the gradual addition of more colour, flashing imagery, additional $3 \mathrm{D}$ objects and moving characters, there may be an impact on mVEP classification accuracy, however the difference in accuracy has not been shown to be significant in this study. The downwards trend in accuracy was expected given the added visual information the user had to attend to and ignore at the same time.

As the game levels become more visually complex, the occipital lobe of the brain which is responsible for vision needs to process a number of different tasks relating to the rich game interface such as 3D objects, motion, colour, flashing imagery and text. The Visual area 5 (V5) also known as the Middle Temporal (MT) area of the occipital lobe is responsible for the processing of motion. The Visual area $1(\mathrm{~V} 1)$ is responsible for the processing of colour and the Visual area 2 (V2) is responsible for the recognition of complex shapes. Active engagement of these areas is likely to distort mVEPs.

Given the increased brain resources required to process increased graphical complexity, it is considered important to take into consideration the 
complexity of the game graphics and their impact on mVEP classification, even though this study has indicated that the complexity does not significantly affect mVEP discrimination performance. The implications of the limited power in the study as a result of the limited number of participants involved and limited number of trials per level assessed are outlined in the following subsection.

The differences in accuracies obtained from the cross level tests vs. the offline tests involving classifier retraining may be caused by a number of factors. For example, the training data which was attained from a completely different game scenario i.e., a basic no graphic interface experienced during the training level (Fig.1) compared to the increasing graphical interfaces experienced by the user as the levels progressed. This suggests that it may be best to set up the classifier and apply it only when stimuli are presented with a similar level of background graphical complexity.

Also, the time difference between the training session and each consecutive game level could be a contributing factor in the decreasing accuracies i.e., the system is using the training data on each consecutive level which was collected up to one hour prior to the level being played.

The sharper decrease attained in the cross level test from level $2(47.7 \%)$ to level $3(41.5 \%)$ may be attributed to the added in game items at the side of the road which consisted of barrel shaped objects and text with scoring information. In the case of the cross level testing, the subjects may have been distracted by this additional content and so the quality of the training data may have been compromised with the additional noise from eye movements, decrease in the subjects' concentration or the attention to details not present during the training data collection.

\subsection{Limitations}

The game graphics presentation levels in this study increased in graphical complexity in a linear fashion starting from basic to more complex i.e., the most complex graphics were presented last in the session for the subjects. This may have confounded results as the subject fatigue and/or electrode gel drying may have impacted on the level results. Results from a limited number of subjects where the level presentation order was randomised suggest that the order of presentation did not influence results, however, to rule out the influence of confounding results in future studies, game levels presentation will be ordered randomly.
To rule out subject fatigue during the sessions it may also be prudent to conduct multiple sessions of shorter duration.

As this was an investigation into graphical complexity, effects on mVEP production and classification we did not provide any real time feedback for the subjects. Feedback on performance during gameplay is critical for game immersion and this limitation may have decreased the participants' interest within the study session. The introduction of feedback into the games is expected to engage the subjects more in game play.

The ANOVA results suggested that the difference in results for each level of complexity were insignificant indicating that the hypothesis that mVEP classification results are decreased with increasing graphical complexity can be rejected. There may be a number of reasons for this finding. It may be that the graphics presented on each consecutive level during the trials were not diverse enough from each other to justify a significant difference. There is significant scope to increase the graphical complexity, bearing in mind the graphical achievements in some of the most advanced and fast paced games on the market today e.g., Call of Duty: Ghosts [22], Gran Turismo 6 [23] and The Last of Us [24]. Also, there were only 30-60 trials per target stimuli for each level which is relatively low for comparative studies and this may have impacted on performance. This, along with the small sample size $(n=10)$ and significant variations across subjects may have impacted on statistical tests. Future studies will involve increasing the graphical complexity, number of trials, sessions conducted and also the number of participants.

\section{CONCLUSION}

In recent years, computer gaming has become a mass market form of entertainment that is enjoyed across demographics. The popularity of computer gaming has fuelled increased interest in novel forms of input and control such as BCI [25].

The results from this study indicate that successful and reliable detection of mVEP responses are possible for 3D video games of varying levels of graphical complexity and that the levels of complexity in game graphics do not adversely affect mVEP discrimination performance. The graphical complexity in this study ranged from minimal to moderate and the results indicate that more complex graphics could impact on mVEP based BCI performance. Further work will involve improving the study design to investigate the impact of more complex graphics on mVEP discrimination and 
developing and testing an mVEP based BCI controlled 3D action video game.

\section{REFERENCES}

[1] J. R. Wolpaw, N. Birbaumer, D. J. McFarland, G. Pfurtscheller, and T. M. Vaughan, "Brain-computer interfaces for communication and control.," Clin. Neurophysiol., vol. 113, no. 6, pp. 767-91, Jun. 2002.

[2] D. Marshall, D. Coyle, S. Member, S. Wilson, and M. Callaghan, "Games, Gameplay, and BCI : The State of the Art," vol. 5, no. 2, pp. 82-99, 2013.

[3] Boris Reuderink; Bram van de Laar; Hayrettin Gurkok; Christian Muhl; Mannes Poel; Anton Nijolt; Dirk Heylen Plass-Oude Bos, Danny, "Brain Computer Interfacing and Games," in Brain Computer Interfaces, Springer London, 2010, pp. 149-178.

[4] S. Lee, Y. Shin, S. Woo, K. Kim, and H. Lee, "Review of Wireless Brain-Computer Interface Systems," 2013.

[5] F. Guo, B. Hong, X. Gao, and S. Gao, "A braincomputer interface using motion-onset visual evoked potential.," J. Neural Eng., vol. 5, no. 4, pp. 477-85, Dec. 2008 .

[6] B. Hong, F. Guo, T. Liu, X. Gao, and S. Gao, "N200speller using motion-onset visual response.," Clin. Neurophysiol., vol. 120, no. 9, pp. 1658-66, Sep. 2009.

[7] D. Marshall, S. Wilson, and D. Coyle, "Motion-Onset Visual Evoked Potentials for Gaming: A pilot study," 24th IET Irish Signals Syst. Conf. (ISSC 2013), pp. 56$56,2013$.

[8] G. Pires, M. Torres, N. Casaleiro, U. Nunes, and M. Castelo-Branco, "Playing Tetris with non-invasive BCI," 2011 IEEE 1st Int. Conf. Serious Games Appl. Heal., pp. 1-6, Nov. 2011.

[9] A. Finke, A. Lenhardt, and H. Ritter, "The MindGame: a P300-based brain-computer interface game.," Neural Netw., vol. 22, no. 9, pp. 1329-33, Nov. 2009.

[10] E. C. Lalor, S. P. Kelly, C. Finucane, R. Burke, R. Smith, R. B. Reilly, and G. McDarby, "Steady-State VEP-Based Brain-Computer Interface Control in an Immersive 3D Gaming Environment," EURASIP J. Adv. Signal Process., vol. 2005, no. 19, pp. 3156-3164, 2005 .
[11] P. Martinez, H. Bakardjian, and A. Cichocki, "Fully online multicommand brain-computer interface with visual neurofeedback using SSVEP paradigm.," Comput. Intell. Neurosci., vol. 2007, no. i, p. 94561, Jan. 2007.

[12] D. Coyle, J. Garcia, A. R. Satti, and T. M. McGinnity, "EEG-based continuous control of a game using a 3 channel motor imagery BCI: BCI game," 2011 IEEE Symp. Comput. Intell. Cogn. Algorithms, Mind, Brain, pp. 1-7, Apr. 2011.

[13] A. S. Royer, A. J. Doud, M. L. Rose, and B. He, "EEG control of a virtual helicopter in 3-dimensional space using intelligent control strategies.," IEEE Trans. Neural Syst. Rehabil. Eng., vol. 18, no. 6, pp. 581-9, Dec. 2010.

[14] "Unity 3D.” Unity Technologies, 2014.

[15] "International 10-20 System." [Online]. Available: http://en.wikipedia.org/wiki/10-20_system_(EEG). [Accessed: 29-May-2014].

[16] "g.tec - g.BSamp Biosignal Amplifier," 2014. [Online]. Available: http://www.gtec.at/Products/Hardware-andAccessories/g.BSamp-Specs-Features. [Accessed: 29May-2014].

[17] "g.Tec - g.GAMMAbox," 2014. [Online]. Available: http://www.gtec.at/Products/Electrodes-and-

Sensors/g.GAMMAsys-Specs-Features. [Accessed: 29May-2014].

[18] "Easycap EEG Cap." [Online]. Available: http://www.easycap.de/easycap/. [Accessed: 29-May2014].

[19] "MATLAB." MathWorks, 2014.

[20] "BioSig Toolbox." [Online]. Available: http://biosig.sourceforge.net/. [Accessed: 06-Jan-2014].

[21] "LIBSVM Toolbox." [Online]. Available: http://www.csie.ntu.edu.tw/ cjlin/libsvm/. [Accessed: 06-Jan-2014].

[22] "Call of Duty: Ghosts." Activision, 2013.

[23] "Gran Turismo 6." Sony Computer Entertainment, 2013.

[24] “The Last of Us.” Naughty Dog, 2013.

[25] A. Nijholt, D. P.-O. Bos, and B. Reuderink, "Turning shortcomings into challenges: Brain-computer interfaces for games," Entertain. Comput., vol. 1, no. 2, pp. 85-94, Apr. 2009. 